TRANSACTIONS OF THE

AMERICAN MATHEMATICAL SOCIETY

Volume 354, Number 9, Pages 3815-3834

S 0002-9947(02)03043-X

Article electronically published on May 7, 2002

\title{
EULER CHARACTERS AND SUBMANIFOLDS OF CONSTANT POSITIVE CURVATURE
}

\author{
JOHN DOUGLAS MOORE
}

\begin{abstract}
This article develops methods for studying the topology of submanifolds of constant positive curvature in Euclidean space. It proves that if $M^{n}$ is an $n$-dimensional compact connected Riemannian submanifold of constant positive curvature in $\mathbb{E}^{2 n-1}$, then $M^{n}$ must be simply connected. It also gives a conformal version of this theorem.
\end{abstract}

\section{INTRODUCTION}

The purpose of this investigation is to explore the relationships which exist between curvature and topology of submanifolds of low codimension in Euclidean space. One of the classical theorems in this direction is due to Chern, Kuiper and Otsuki, and states that there is no compact $n$-dimensional submanifold of nonpositive sectional curvatures in $\mathbb{E}^{2 n-1}$. Indeed, if such a submanifold were to exist, one could take a large sphere at infinity and contract it until a first point of tangency occurred. An algebraic argument would then show that at least one of the sectional curvatures at this point must be positive. (See [8], Volume 2, page 29, Corollary 2.8.) This is an extension to higher dimensions of the well-known fact that a smooth surface in $\mathbb{E}^{3}$ of nonpositive curvature cannot be compact.

It is natural to suspect that there are significant topological restrictions on compact submanifolds of positive sectional curvatures which admit isometric immersions in low codimensions. As a first step in this direction, we presented a Morse theory argument in 12 which showed that if $n \geq 3$, a compact $n$-dimensional submanifold of $\mathbb{E}^{n+2}$ with positive sectional curvatures must be homeomorphic to a sphere. Since the method employed seems to break down in higher codimensions, it is of interest to develop alternate techniques. This article utilizes the theory of Cheeger-Simons characters to give a contribution in higher codimensions to the constant curvature case:

Theorem. Suppose that $M^{n}$ is an n-dimensional compact connected Riemannian manifold of constant positive curvature. If $M$ possesses an isometric immersion into $\mathbb{E}^{2 n-1}$ or a conformal immersion into $\mathbb{E}^{2 n-3}$, then $M$ is simply connected, and hence isometric to the standard constant curvature $n$-sphere.

This theorem has a global geometric character. Indeed, it was proven by Whitney that any compact smooth $n$-manifold can be immersed in $\mathbb{E}^{2 n-1}$. On the other hand, there is a rich theory of locally defined $n$-dimensional submanifolds of constant positive curvature in $\mathbb{E}^{2 n-1}$. Indeed, it was shown in [1] that there are two

Received by the editors March 28, 2001.

2000 Mathematics Subject Classification. Primary 53C40; Secondary 57R20.

(C) 2002 American Mathematical Society 
local types of such submanifolds, those consisting of "nonumbilic points" and those consisting of "weak umbilics". Moreover, it can be shown that the ones containing nonumbilic points depend upon $n(n-1)$ functions of a single variable, in the sense of Cartan-Kähler theory.

Corollary. The only compact connected $n$-dimensional space form which admits an isometric immersion into $\mathbb{E}^{2 n-1}$ or a conformal immersion into $\mathbb{E}^{2 n-3}$ is the $n$-sphere with its standard metric.

To obtain the Corollary from the Theorem, we need to rule out space forms with zero or negative curvature. In the case of isometric immersions, these are eliminated by the previously mentioned theorem of Chern, Kuiper and Otsuki. In the case of conformal immersions, they are eliminated by results first presented in [13], which we will review in $\S 4$ of this article.

We recall that one of the first applications of the Chern-Simons invariants, constructed from the transgressed Pontrjagin forms, was a proof that the standard constant curvature real projective three-space admits no conformal immersions into $\mathbb{E}^{4}$. Exactly the same argument shows that the only three-dimensional compact Riemannian manifold of constant positive curvature which conformally immerses in $\mathbb{E}^{4}$ is the standard three-sphere. These invariants were also used to show that many $n$-dimensional constant curvature lens spaces do not admit conformal immersions into Euclidean space of dimension roughly $(3 / 2) n[9]$. On the other hand, via Morse theory, it was possible to show that the only $n$-dimensional positively curved space form which immerses isometrically into $\mathbb{E}^{[(3 / 2) n]}$ or conformally into $\mathbb{E}^{[(3 / 2) n-1]}$, where $[x]$ denotes the largest integer $\leq x$, is the standard $n$-sphere with constant curvature metric. (See Proposition 4, page 455 in [11] and the Corollary, page 90 in 10 .)

The proof of the Theorem presented here is based upon Chern-Simons invariants, reformulated as the theory of differential characters by Cheeger and Simons, but our starting point is the transgressed Euler form instead of the transgressed Pontrjagin forms. In addition, we utilize techniques we have developed for studying the local structure of submanifolds of constant positive curvature. After preliminaries in $\S 2$, we present a proof of the Theorem in the case of isometric immersions in $\S 3$, except for a technical lemma on smoothness of a certain decomposition of the normal bundle, which we present in $\S 6$. It is hoped that this lemma will have other applications to the global geometry of submanifolds. The argument for the case of conformal immersions is presented in $\S 4$ and $\S 5$.

The author thanks Jean-Marie Morvan and Vincent Borrelli for pointing out a mistake in an earlier approach to proving the above theorem.

\section{Structure of Submanifolds of CONSTANT POSITIVE CURVATURE}

Let $M^{n}$ be an $n$-dimensional compact connected Riemannian manifold of constant positive curvature, normalized to be one, $f: M^{n} \rightarrow \mathbb{E}^{N}$ an isometric immersion. Then the second fundamental form of $f$,

$$
\alpha: T_{p} M \times T_{p} M \rightarrow N_{p} M,
$$

satisfies the Gauss equation

$$
\langle\alpha(x, z), \alpha(y, w)\rangle-\langle\alpha(x, w), \alpha(y, z)\rangle=\langle x, z\rangle\langle y, w\rangle-\langle x, w\rangle\langle y, z\rangle,
$$


for $x, y, z, w \in T_{p} M$. A theorem of O'Neill [16] states that if $N \leq 2 n-2$, there is a unique unit-length vector $e_{n+1}(p) \in N_{p} M$ such that

$$
\left\langle\alpha(x, y), e_{n+1}(p)\right\rangle=\langle x, y\rangle, \quad \text { for } x, y \in T_{p} M .
$$

The case where $N=2 n-1$ is analyzed in [11. In this case, we say that $p$ is a weak umbilic if the second fundamental form at $p$ satisfies (1) for some choice of $e_{n+1}(p) \in$ $N_{p} M$; otherwise $p$ is called a nonumbilic. If $M$ were noncompact, both possibilities could occur, but Theorem 3 of [11] shows that there are no nonumbilics when $M$ is compact, by an argument which generalizes the standard proof of Liebmann's theorem that the constant curvature two-sphere $S^{2}$ is rigid in $\mathbb{E}^{3}$.

Thus, under our compactness assumption, we have a well-defined normal section $p \mapsto e_{n+1}(p)$ such that $e_{n+1}(p)$ satisfies (1) at each $p \in M$. This section is easily seen to be continuous, but the proof that it is smooth is surprisingly subtle. We will present the argument for smoothness in $\S 6$. This extends a much simpler argument for smoothness in the codimension two case that was presented in [14], together with an application to extendability in codimension two.

Assuming that the section $p \mapsto e_{n+1}(p)$ is smooth, we can divide the normal bundle $N M$ into a direct sum $N M=L \oplus E^{\perp}$, where $L$ is the line bundle generated by $e_{n+1}$ and $E^{\perp}$ is its orthogonal complement. We let $E$ denote the direct sum $T M \oplus L$; so $E \oplus E^{\perp}$ is a direct sum decomposition of the trivial $N$-plane bundle over $M$. The second fundamental form divides into two orthogonal components

$$
\alpha(x, y)=\langle x, y\rangle e_{n+1}(p)+\beta(x, y)
$$

where the $E^{\perp}$-component $\beta$ satisfies

$$
\langle\beta(x, z), \beta(y, w)\rangle-\langle\beta(x, w), \beta(y, z)\rangle=0, \quad \text { for } x, y, z, w \in T_{p} M .
$$

In the terminology of [10], a vector-valued symmetric bilinear form $\beta$ that satisfies (3) is called flat.

We utilize the index conventions:

$$
1 \leq a, b, c \leq n ; \quad 1 \leq i, j, k \leq n+1 ; \quad 1 \leq I, J, K \leq N ; \quad n+2 \leq \lambda, \mu, \nu \leq N .
$$

Working locally over a suitable open subset $U$ of $M$, we choose a moving orthonormal frame $\left(e_{1}, \ldots, e_{n}, e_{n+1}\right)$ for $E$ so that the last frame field agrees with the section of $L$ chosen before. We supplement this with a moving orthonormal frame $\left(e_{n+2}, \ldots, e_{N}\right)$ for $E^{\perp}$. The connection forms

$$
\omega_{I J}=\left\langle e_{I}, \nabla e_{J}\right\rangle,
$$

defined via the standard flat connection $\nabla$ on the trivial bundle $E \oplus E^{\perp}=M \times$ $\mathbb{E}^{N}$, satisfy the skew-symmetry condition $\omega_{J I}=-\omega_{I J}$ together with the Cartan structure equations

$$
d \omega_{I J}=-\sum_{K} \omega_{I K} \wedge \omega_{K J} .
$$

Moreover, if $\theta_{1}, \ldots, \theta_{n}$ denote the canonical one-forms defined by $\theta_{a}\left(e_{b}\right)=\delta_{a b}$, and $\theta_{n+1}=\theta_{\lambda}=0$,

$$
d \theta_{I}=-\sum_{J} \omega_{I J} \wedge \theta_{J}
$$


The matrix $\left(\omega_{I J}\right)$ of connection forms divides up into blocks

$$
\left(\begin{array}{cc}
\omega_{i j} & \omega_{i \mu} \\
\omega_{\lambda j} & \omega_{\lambda \mu}
\end{array}\right)
$$

where the upper left $(n+1) \times(n+1)$ block represents the induced connection in $E$ and the lower right block represents the induced connection in $E^{\perp}$.

The upper left block divides further into blocks

$$
\left(\begin{array}{cc}
\omega_{a b} & \omega_{a, n+1} \\
\omega_{n+1, b} & 0
\end{array}\right)
$$

with the upper left $n \times n$ block representing the Levi-Civita connection on $T M^{n}$ and the other nonzero blocks representing the second fundamental form of $M^{n}$ in E. According to equation (2),

$$
\left\langle\alpha, e_{n+1}\right\rangle=\sum_{a} \omega_{n+1, a} \otimes \theta_{a}=\sum_{a} \theta_{a} \otimes \theta_{a}
$$

and hence $\omega_{n+1, a}=\theta_{a}$. The curvature forms $\Omega_{i j}$ for the connection in $E$ are defined by

$$
\Omega_{i j}=d \omega_{i j}+\sum_{k} \omega_{i k} \wedge \omega_{k j}
$$

Using the structure equations for $M$,

$$
d \omega_{a b}=-\sum_{c} \omega_{a c} \wedge \omega_{c b}+\theta_{a} \wedge \theta_{b}, \quad d \theta_{a}=-\sum_{b} \omega_{a b} \wedge \theta_{b},
$$

and the fact that $\omega_{n+1, a}=\theta_{a}$, we easily find that $\Omega_{i j}=0$. We thus conclude that the curvature of the geometric bundle $E$ is zero.

If $M^{n}$ is oriented, we can define a Gauss map

$$
g: M^{n} \longrightarrow G_{n+1}^{+}\left(\mathbb{E}^{N}\right)=\left\{\text { oriented }(n+1) \text {-planes in } \mathbb{E}^{N}\right\},
$$

by letting $g(p)$ be the subspace of $\mathbb{E}^{N}$ generated by $e_{1}, \ldots, e_{n}, e_{n+1}$. This map is covered by a vector bundle map

$$
\tilde{g}: E \longrightarrow E_{\infty}=\text { universal bundle over } G_{n+1}^{+}\left(\mathbb{E}^{N}\right),
$$

the fiber of $E_{\infty}$ over a given oriented subspace of $\mathbb{E}^{N}$ being that subspace.

Lemma. The differential of the Gauss map $g$ at any point has rank at most $n-2$.

Proof. We first extend the $E^{\perp}$-component $\beta$ of the second fundamental form to a bilinear map

$$
\tilde{\beta}: T_{p} M \times E_{p} \longrightarrow E_{p}^{\perp} \quad \text { by } \quad \tilde{\beta}\left(x, \sum a_{i} e_{i}\right)=\sum_{\lambda i} a_{i} e_{\lambda} \omega_{\lambda i}(x) .
$$

Differentiating the equation

$$
\omega_{n+1, a}=\theta_{a}
$$

and applying the structure equations (15), we find that

$$
\sum_{\lambda} \omega_{\lambda, n+1} \wedge \omega_{\lambda, a}=0
$$

This together with (3) implies that

$$
\langle\tilde{\beta}(x, z), \tilde{\beta}(y, w)\rangle-\langle\tilde{\beta}(x, w), \tilde{\beta}(y, z)\rangle=0, \quad \text { for } x, y \in T_{p} M \text { and } z, w \in E_{p} .
$$


We now apply a slight modification of an argument from the theory of flat bilinear forms (which is described in [11]). For $z \in E_{p}$, we define

$$
\tilde{\beta}(z): T_{p} M \longrightarrow E_{p}^{\perp} \quad \text { by } \quad \tilde{\beta}(z)(x)=\tilde{\beta}(x, z)
$$

and let $\mu$ denote the maximum dimension of the rank of $\tilde{\beta}(z)$ as $z$ ranges over $E_{p}$. We say that an element $z \in E_{p}$ is regular for $\tilde{\beta}$ if the rank of $\tilde{\beta}(z)$ is $\mu$. Although the rank of $\tilde{\beta}(z)$ may be less than $\mu$ for particular values of $z$, regular elements form an open dense subset of $E_{p}$. Clearly if $z$ is regular,

$$
\operatorname{dim}(N(\tilde{\beta}(z)))=n-\mu \geq \operatorname{dim}\left(T_{p} M\right)-\left(\operatorname{dim}\left(N_{p} M\right)-1\right)=n-(N-(n+1)) .
$$

However, the space we need for our argument is

$$
N(\tilde{\beta})_{p}=\bigcap\left\{N(\tilde{\beta}(z)): z \in E_{p}\right\}=\left\{v \in T_{p} M: \omega_{\lambda i}(v)=0\right\} .
$$

To analyze this space, we recall the argument for the Lemma in [10]. Suppose that $z$ is a regular element of $E_{p}$ and $n \in N(\tilde{\beta}(z))$. It follows from (6) that

$$
\langle\tilde{\beta}(n, w), \tilde{\beta}(x, z)\rangle=0, \quad \text { for all } x \in T_{p} M, w \in E_{p} .
$$

On the other hand, the lower semicontinuous function

$$
t \mapsto \operatorname{rank} \text { of } \tilde{\beta}(z+t w)
$$

must equal $\mu$ in a neighborhood of zero, and when $t$ is sufficiently near zero, $\tilde{\beta}(z+$ $t w)\left(T_{p} M\right)$ is a continuously varying $\mu$-dimensional space. Thus

$$
\tilde{\beta}(n, z+t w)=t \beta(n, w) \quad \Rightarrow \quad \beta(n, w) \in \beta(z+t w)\left(T_{p} M\right) \text { for small } t,
$$

and by continuity $\beta(n, w) \in \beta(z)\left(T_{p} M\right)$. Comparison with (7) now shows that $\tilde{\beta}(n, w)=0$ and thus $n \in N(\tilde{\beta})_{p}$. Hence $N(\tilde{\beta}(z))=N(\tilde{\beta})_{p}$ and

$$
\operatorname{dim}\left(N(\tilde{\beta})_{p}\right)=\operatorname{dim}(N(\tilde{\beta}(z))) \geq 2 n-N+1
$$

We define an upper semicontinuous function $\nu: M \rightarrow Z$ by $\nu(p)=\operatorname{dim} N(\tilde{\beta})_{p}$, and let

$$
U_{k}=\{p \in M: \nu(p) \leq k\},
$$

thereby obtaining an increasing filtration of $M$ by open sets

$$
U_{0} \subset U_{1} \subset \cdots \subset U_{k} \subset \cdots \subset U_{n}=M \text {. }
$$

On the open set $U_{k}-\overline{U_{k-1}}, p \mapsto N(\tilde{\beta})_{p}$ is a distribution of constant rank $k$, and it follows from (4) that

$$
d \omega_{\lambda i} \in \text { ideal generated by the } \omega_{\mu j} \text { 's, }
$$

and hence this distribution is involutive. Thus the maximal integral submanifolds form a foliation $\mathcal{F}$ of $U_{k}-\overline{U_{k-1}}$ with $k$-dimensional leaves, which we call the umbilic foliation.

Since the restriction of $\omega_{\lambda i}$ to each leaf of $\mathcal{F}$ is zero, it follows that the subbundles $E$ and $E^{\perp}$ of the trivial bundle $M \times \mathbb{E}^{N}$ are preserved by parallel transport along the leaves. Hence the restriction of the Gauss map $g$ to each leaf is constant. Therefore, on the open set $U_{k}-\overline{U_{k-1}}$, the differential of the Gauss map has rank at most $n-k$. Since $k \geq 2 n-N+1$ and $N \leq 2 n-1$, the differential of the Gauss map has rank at most $N-(n+1) \leq n-2$ on

$$
\bigcup\left\{U_{k}-\overline{U_{k-1}}: k \geq 2 n-N+1\right\} \text {. }
$$


This is an open dense subset of $M$; so we conclude that the differential of $g$ has rank $\leq n-2$ at every point of $M$, and the Lemma is proven.

\section{EULER CHARACTERS}

We next review the theory of differential characters, as formulated by Cheeger and Simons 3]. For our argument, it will be convenient to modify the definition slightly.

Suppose that the smooth manifold $M$ is embedded in $\mathbb{E}^{N}$, for some $N$. Let $\mathcal{D}_{k}(M)$ denote the space of $k$-dimensional currents on $M$, as described for example in Chapter 4 of [7] and let $S_{k}(M)$ denote the space of singular $k$-chains on $M$. Recall that each element of $S_{k}(M)$ is simply a smooth map from the standard $k$-simplex into $M$. If $\sigma \in S_{k}(M)$, let $i(\sigma) \in \mathcal{D}_{k}(M)$ be the current defined by integration over $\sigma$. It follows from the theorem of Stokes that $i$ commutes with the boundary operator, thereby defining a chain map between chain complexes,

$$
i_{*}: S_{*}(M) \longrightarrow \mathcal{D}_{*}(M) .
$$

The kernel of this chain map is a subcomplex $S_{*}^{0}(M)$ consisting of what we might call degenerate smooth singular simplices.

We claim that $S_{*}^{0}(M)$ is acyclic. To see this, we give $M$ a smooth simplicial decomposition and let $M^{k}$ denote the $k$-skeleton of $M$. It suffices to show that any cycle $\sigma \in S_{k}^{0}\left(M^{k}\right)$ is a boundary. But degeneracy implies that $\sigma$ misses at least one point from each $k$-simplex and is therefore chain homotopic to an element in $S_{k}^{0}\left(M^{k-1}\right)=S_{k}\left(M^{k-1}\right)$. Since the $k$-dimensional homology of $M^{k-1}$ vanishes, it follows that $\sigma$ is a boundary and our claim is established.

It follows that the quotient complex $C_{*}(M)=S_{*}(M) / S_{*}^{0}(M)$ calculates the cohomology of $M$. The advantage of this complex over the complex of smooth singular chains is that we can regard it as a subcomplex of $\mathcal{D}_{*}(M)$ and in particular, if $\sigma \in C_{k}(M)$ and the integral over $\sigma$ of every $k$-form vanishes, then $\sigma$ must be zero.

We let $Z_{k}(M)$ denote the space of $k$-dimensional cycles, the elements $\sigma \in C_{k}(M)$ such that $\partial \sigma=0$, and let $\Omega_{0}^{k+1}(M)$ denote the space of smooth $(k+1)$-forms on $M$ that have integral periods. We can then define the space of $k$-dimensional differential characters on $M$ by

$$
\begin{aligned}
& \widehat{H}^{k}(M, \mathbb{R} / \mathbb{Z})=\left\{f \in \operatorname{Hom}\left(Z_{k}(M), \mathbb{R}\right): \text { there exists } \omega \in \Omega_{0}^{k+1}(M)\right. \\
& \text { such that } \left.f(\partial \sigma)=\bmod \mathbb{Z} \text { reduction of } \int_{\sigma} \omega \text { for } \sigma \in C_{k+1}(M)\right\} .
\end{aligned}
$$

Note that these characters are functorial; that is, a smooth map $f: M \rightarrow N$ induces a linear map

$$
f^{*}: \widehat{H}^{k}(N, \mathbb{R} / \mathbb{Z}) \longrightarrow \widehat{H}^{k}(M, \mathbb{R} / \mathbb{Z}) .
$$

This definition is identical to that presented in [3], except that we have replaced smooth singular cycles with elements of $C_{*}(M)$.

A special case is that of the Euler character $\hat{\chi}(\nabla)$ of a geometric vector bundle $(E,\langle\cdot, \cdot\rangle, \nabla)$. In our context, a geometric vector bundle is simply an oriented smooth vector bundle $E$ together with a fiber metric $\langle\cdot, \cdot\rangle$ and a metric connection $\nabla$. The Euler character is most easily defined on the universal bundle $E_{\infty}$ over $G_{n+1}^{+}\left(\mathbb{E}^{N}\right)$ with its standard metric and universal connection $\nabla_{\infty}$, and then on more general 
geometric bundles by naturality. Note that $G_{n+1}^{+}\left(\mathbb{E}^{N}\right)$ can be identified with the submanifold of decomposable vectors in the Euclidean space $\Lambda^{n+1} \mathbb{E}^{N}$.

Suppose that $n$ is odd. Since the odd-dimensional homology of the Grassmannian $G_{n+1}^{+}\left(\mathbb{E}^{N}\right)$ consists of $\mathbb{Z}_{2}$ torsion,

$$
\tau \in Z_{n}\left(G_{n+1}^{+}\left(\mathbb{E}^{N}\right)\right) \quad \Rightarrow \quad \text { either } \tau \text { or } 2 \tau \text { bounds. }
$$

If $\tau=\partial \sigma$, we define the Euler character $\hat{\chi}\left(\nabla_{\infty}\right)$ by

$$
\hat{\chi}\left(\nabla_{\infty}\right)(\tau)=\int_{\sigma} e\left(\nabla_{\infty}\right) \quad(\bmod \mathbb{Z}) .
$$

If $2 \tau=\partial \sigma$, we set

$$
\hat{\chi}\left(\nabla_{\infty}\right)(\tau)=\frac{1}{2}\left[\int_{\sigma} e\left(\nabla_{\infty}\right)-\left\langle u\left(E_{\infty}\right), \sigma\right\rangle\right] \quad(\bmod \mathbb{Z}),
$$

where $u\left(E_{\infty}\right)$ is an integral cocycle representing the universal integral Euler class, the integral Euler class of $E_{\infty}$. It is readily verified that $\hat{\chi}\left(\nabla_{\infty}\right)(\tau)$ does not depend upon the choice of either $\sigma$ or $u\left(E_{\infty}\right)$.

According to a theorem of Narasimhan and Ramanan [15, any geometric bundle can be obtained by pulling back from the universal bundle over $G_{n+1}^{+}\left(\mathbb{E}^{N}\right)$ for sufficiently large $N$. In other words, if $(E,\langle\cdot, \cdot\rangle, \nabla)$ is a geometric bundle, there is a smooth classifying map $g: M \rightarrow G_{n+1}^{+}\left(\mathbb{E}^{N}\right)$ for $N$ large, so that $E=g^{*} E_{\infty}$ and $\langle\cdot, \cdot\rangle$ and $\nabla$ are the fiber metric and connection induced by $g$ from the metric and connection on the universal bundle. We can then define the Euler character of the geometric bundle $(E,\langle\cdot, \cdot\rangle, \nabla)$ by $\hat{\chi}(\nabla)=g^{*} \hat{\chi}\left(\nabla_{\infty}\right)$. The proof given in 3 that $\hat{\chi}(\nabla)$ is independent of the choice of classifying map $g$ carries over immediately to the case where the smooth singular cycles are replaced with elements of $C_{*}(M)$.

As a particular case, we can take $M$ to be the total space of the unit-sphere bundle corresponding to the universal bundle $E_{\infty}$ over $G_{n+1}^{+}\left(\mathbb{E}^{N}\right)$,

$$
M=\frac{S O(N)}{S O(n) \times 1 \times S O(N-n-1)} \quad \text { as a homogeneous space. }
$$

Over $M$ we can consider the pullback $\pi^{*} E_{\infty}$ of the universal bundle, where $\pi: M \rightarrow$ $G_{n+1}^{+}\left(\mathbb{E}^{N}\right)$ is the projection. The odd-dimensional homology of $M$ still consists of $\mathbb{Z}_{2}$ torsion; so formulae [8] and (9) can be used with $e\left(\nabla_{\infty}\right)$ replaced by $\pi^{*} e\left(\nabla_{\infty}\right)$ and $u\left(E_{\infty}\right)$ replaced by a representative for the integral Euler class of $\pi^{*} E_{\infty}$. But this integral Euler class is zero, while

$$
\pi^{*} e\left(\nabla_{\infty}\right)=d\left[T e\left(\nabla_{\infty}\right)\right],
$$

where $T e\left(\nabla_{\infty}\right)$ is the transgressed Euler form, or geodesic curvature form, constructed by Chern in his intrinsic proof of the Gauss-Bonnet formula 4]. Thus it follows from Stokes's theorem that the corresponding Euler character on $M$ is defined by the simple formula

$$
\hat{\chi}\left(\pi^{*} \nabla_{\infty}\right)(\tau)=\int_{\tau} \operatorname{Te}\left(\nabla_{\infty}\right) \quad(\bmod \mathbb{Z}) .
$$

More generally, suppose that $(E,\langle\cdot, \cdot\rangle, \nabla)$ is any geometric vector bundle over a general smooth manifold $M$ which possesses a smooth unit-length section $s: M \rightarrow$ $E$. If the geometric vector bundle possesses a classifying map $g: M \rightarrow G_{n+1}^{+}\left(\mathbb{E}^{N}\right)$, 
the section $s$ defines a lifting of $g$ to a map

Moreover,

$$
\tilde{g}: M \longrightarrow \frac{S O(N)}{S O(n) \times 1 \times S O(N-n-1)} .
$$

$$
\tilde{g}^{*}\left[\operatorname{Te}\left(\nabla_{\infty}\right)\right]=s^{*}[T e(\nabla)],
$$

where $T e(\nabla)$ is the transgressed Euler form on the unit sphere bundle associated to $E$. In this case, naturality implies that the Euler character of $(E,\langle\cdot, \cdot\rangle, \nabla)$ is given by

$$
\hat{\chi}(\nabla)(\tau)=\int_{\tau} s^{*} T e(\nabla) \quad(\bmod \mathbb{Z}) .
$$

Proof of the Theorem in the case of isometric immersions. Suppose first that the dimension $n$ of $M$ is odd. Then $M$ is oriented by Synge's theorem and we can apply (10) to the geometric bundle $(E,\langle\cdot, \cdot\rangle, \nabla)$ over the space form $M$ which was constructed in $\S 2$. If $\mu_{M}$ is a cycle representing the fundamental class of $M$ and $s=e_{n+1}: M \rightarrow E$ is the smooth unit-length section of $E$ described in $\S 2$, a direct calculation shows that

$$
\hat{\chi}(\nabla)\left(\mu_{M}\right)=\int_{M} s^{*} T e(\nabla)=\frac{1}{m} \quad(\bmod \mathbb{Z}),
$$

where $m$ is the order of the fundamental group $\pi_{1}(M)$. Indeed, in the special case in which $M=S^{n}$, the bundle $E$ is trivial, $T e(\nabla)$ is the volume form on the fiber, normalized so that the fiber has unit volume, while $s: S^{n} \rightarrow S^{n}$ is simply the identity map. In this case, $s^{*} T e(\nabla)$ is just the volume form on $S^{n}$. For a general elliptic space form $M$, it follows by naturality that $s^{*} T e(\nabla)$ must also be the volume form on $M$, and hence (11) follows from the fact that the normalized volume of $M$ is $1 / m$, where $m$ is the order of $\pi_{1}(M)$.

However, if $M$ is not simply connected, the Lemma from $\S 2$ yields a conflicting result. To see this, we use the special Gauss map $g$ provided by the isometric immersion. Suppose that $\mu_{M}$ is the $n$-dimensional cycle representing the fundamental class $\left[\mu_{M}\right]$ of $M$. The Lemma implies that the differential of $g$ has rank $\leq n-2$ at every point of $M$ and hence $g_{*}\left(\mu_{M}\right)$ is a current which yields zero when applied to any differential form, and must therefore be zero. It therefore follows from (8) that

$$
\hat{\chi}(\nabla)\left(\mu_{M}\right)=\hat{\chi}\left(\nabla_{\infty}\right)\left(g_{*}\left(\mu_{M}\right)\right)=0 \quad(\bmod \mathbb{Z}) .
$$

This contradicts the calculation that this residue class is nonzero, unless $M$ is simply connected, thus establishing the statement of the Theorem in the case of isometric immersions when $n$ is odd.

If $n$ is even, the preceding argument does not apply. However, in this case, Synge's theorem implies that there are only two possible compact space forms of curvature one, $S^{n}$ and the real projective space $P^{n}$; so we only need to eliminate $P^{n}$. The case $n=2$ is covered by a well-known theorem from classical surface theory, which asserts that a positively curved compact immersed surface is imbedded and bounds a convex body, and must therefore be simply connected. If $n \geq 4$, we can reduce to the odd-dimensional case by considering the immersion $F \circ \iota$, where $\iota: P^{n-1} \rightarrow P^{n}$ is a totally geodesic imbedding. Let $E=T P^{n-1} \oplus L$, an evendimensional bundle which is easily seen to have nonvanishing Euler character by the same calculation described before. Moreover, our previous argument shows that the Gauss map $g: P^{n-1} \rightarrow G_{n}^{+}\left(\mathbb{E}^{N}\right)$ has differential which has rank $\leq(n-1)-1$ at each 
point, and hence the above argument shows once again that the Euler character is zero, yielding a contradiction. This finishes the proof except for the smoothness of $e_{n+1}$, which will be treated in $\S 6$.

\section{Conformally flat SUBmanifolds}

We next review the basic theory of conformal immersions of space forms, following [10] and [13].

We consider Minkowski space-time $\mathbb{L}^{N+2}$, with its standard coordinate system $\left(x_{1}, \ldots, x_{N+1}, t\right)$ and its standard Lorentz metric

$$
\langle\cdot, \cdot\rangle=\left(d x_{1}\right)^{2}+\cdots+\left(d x_{N+1}\right)^{2}-(d t)^{2} .
$$

Let $P^{N+1}$ denote the projective space of lines through the origin in $\mathbb{L}^{N+2}, \pi$ : $\mathbb{L}^{N+2}-\{0\} \rightarrow P^{N+1}$ the projection of a point onto the line that contains it. Let $C^{N+1}$ denote the light cone defined by the equation

$$
x_{1}^{2}+\cdots+x_{N+1}^{2}-t^{2}=0,
$$

$C_{+}^{N+1}$ the intersection of this light cone with the half-space $t>0$. The Lorentz group $O(N+1,1)$ acts as isometries on Minkowski space-time and induces an action via conformal transformations on the $N$-sphere $S^{N}=\pi\left(C^{N+1}-\{0\}\right)$ when it is given the standard conformal structure: A metric $g$ on $S^{N}$ belongs to this conformal structure if it is induced from the Lorentz metric on $\mathbb{L}^{N+2}$ by some smooth section

$$
S^{N} \longrightarrow C^{N+1} \subset \mathbb{L}^{N+2} \quad \text { of the projection } \quad \pi: C^{N+1}-\{0\} \longrightarrow S^{N} .
$$

If $u$ is a nonzero timelike vector in $\mathbb{L}^{N+2}$ and $c \neq 0$, the hyperplane consisting of the points $p \in \mathbb{L}^{N+2}$ that satisfy the equation $p \cdot u=c$ will intersect the cone $C^{N+1}$ in the image of such a section; in this case, the metric induced on $S^{N}$ has constant positive curvature. On the other hand, if $u$ is a nonzero null vector and $c \neq 0$, the hyperplane $p \cdot u=c$ will intersect the cone in a submanifold which is isometric to Euclidean space $\mathbb{E}^{N}$ when it is given the induced metric. Projection along lightlike rays in the light cone yields the standard conformal diffeomorphism from $\mathbb{E}^{N}$ to $S^{N}$ minus a point.

A smooth immersion $f: M^{n} \rightarrow \mathbb{E}^{N} \subset S^{N}$ from a smooth $n$-dimensional manifold $M^{n}$ can be lifted in many ways to a spacelike immersion

$$
\tilde{f}: M^{n} \longrightarrow C_{+}^{N+1} \subset \mathbb{L}^{N+2},
$$

as illustrated in Figure 11 Given any such lifting, the Lorentz metric on $\mathbb{L}^{N+2}$ induces a Riemannian metric on $M$. Two different liftings $\tilde{f}_{1}$ and $\tilde{f}_{2}=e^{\lambda} f_{1}$ induce conformally related metrics $\langle\cdot, \cdot\rangle_{1}$ and $\langle\cdot, \cdot\rangle_{2}=e^{2 \lambda}\langle\cdot, \cdot\rangle_{1}$, and hence the immersion $f$ induces a conformal equivalence class of Riemannian metrics on $M$.

Suppose now that $M$ is given a distinguished Riemannian metric within this conformal equivalence class and that $\tilde{f}$ is a lifting which is isometric for this metric. If $p \in M$, we let $N_{p} M$ denote the normal space to $\tilde{f}$ at $p$, so that

$$
T_{\tilde{f}(p)} \mathbb{L}^{N+2} \cong T_{p} M \oplus N_{p} M
$$

We recall that the second fundamental form $\alpha: T_{p} M \times T_{p} M \rightarrow N_{p} M$ of $\tilde{f}$ at $p$ is defined by

$$
\alpha\left(\gamma^{\prime}(0), \gamma^{\prime}(0)\right)=(\tilde{f} \circ \gamma)^{\prime \prime}(0)^{\perp}=\text { normal component of }(\tilde{f} \circ \gamma)^{\prime \prime}(0),
$$



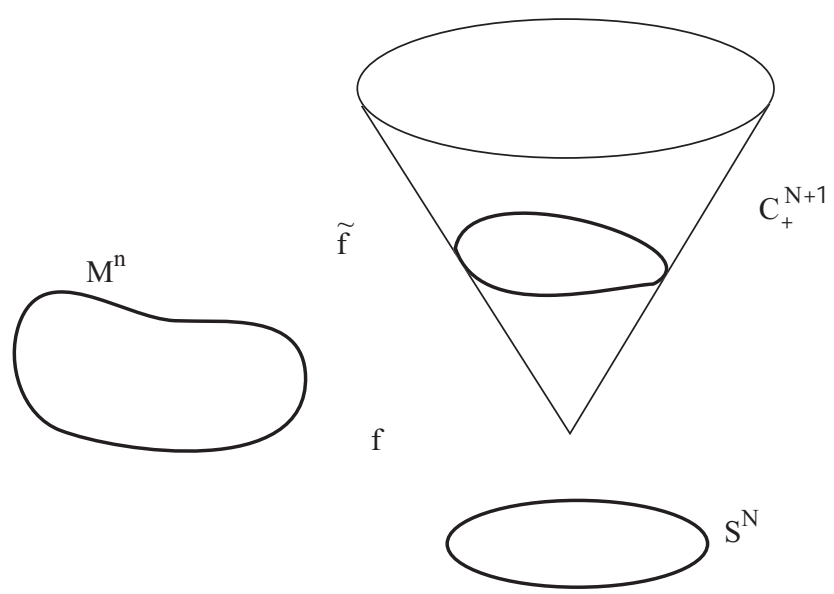

Figure 1. A conformal immersion of a space form into $S^{N}$ can be lifted to an isometric immersion in the forward half $C_{+}^{N+1}$ of the light cone in $\mathbb{L}^{N+2}$.

whenever $\gamma:(-\epsilon, \epsilon) \rightarrow M$ is a smooth curve with $\gamma(0)=p$. Since $\tilde{f}(p)$ is perpendicular to the tangent space to the light cone, it is perpendicular to $\tilde{f}(M)$ at $\tilde{f}(p)$. Differentiating the identity

$$
\langle(\tilde{f} \circ \gamma)(t),(\tilde{f} \circ \gamma)(t)\rangle=0
$$

twice with respect to $t$, we obtain

$$
\left\langle(\tilde{f} \circ \gamma)^{\prime \prime}(0),(\tilde{f} \circ \gamma)(0)\right\rangle=-\left\langle(\tilde{f} \circ \gamma)^{\prime}(0),(\tilde{f} \circ \gamma)^{\prime}(0)\right\rangle,
$$

from which it follows that

$$
\langle\alpha(x, x), \tilde{f}(p)\rangle=-\langle x, x\rangle, \quad \text { for } x \in T_{p} M .
$$

By polarization, we conclude that

$$
\langle\alpha(x, y), \tilde{f}(p)\rangle=-\langle x, y\rangle, \quad \text { for } x, y \in T_{p} M .
$$

We now specialize to the case where the distinguished metric on $M$ is locally conformally flat. In this case we can choose a neighborhood $U$ of $p$ and a real-valued function $\lambda: U \rightarrow \mathbb{R}$ such that

$$
\tilde{f}_{0}=e^{\lambda} \tilde{f}: U \longrightarrow C_{+}^{N+1} \subset \mathbb{L}^{N+2}
$$

induces the flat metric on $U$. Thus the second fundamental form $\alpha_{0}$ of $\tilde{f}_{0}$ must satisfy the Gauss equation

$$
\left\langle\alpha_{0}(x, z), \alpha_{0}(y, w)\right\rangle-\left\langle\alpha_{0}(x, w), \alpha_{0}(y, z)\right\rangle=0, \quad \text { for } x, y, z, w \in T_{p} M,
$$

which expresses the fact that $\alpha_{0}$ is a flat symmetric bilinear form. On the other hand, the second fundamental form of $\tilde{f}_{0}$ is simply the projection of

$$
\begin{aligned}
\left(e^{\lambda}(\tilde{f} \circ \gamma)\right)^{\prime \prime} & (0)=e^{\lambda(0)}\left[(\tilde{f} \circ \gamma)^{\prime \prime}(0)\right. \\
& \left.\left.+2 \lambda^{\prime}(0)(\tilde{f} \circ \gamma)^{\prime}(0)+\left(\lambda^{\prime \prime}(0)+\lambda^{\prime}(0)\right)^{2}\right)(\tilde{f} \circ \gamma)(0)\right]
\end{aligned}
$$


into the normal space to $\tilde{f}_{0}$. We can choose $\lambda$ so that $d \lambda(p)=0$; then the tangent and normal spaces to $\tilde{f}$ and $\tilde{f}_{0}$ agree, and the normal component of the second derivative simplifies to yield

$$
\alpha_{0}=e^{\lambda} \alpha+\psi_{0} \tilde{f}
$$

where $\psi_{0}: T_{p} M \times T_{p} M \rightarrow \mathbb{R}$ is a real-valued symmetric bilinear form. Solving for $\alpha$, we obtain

$$
\alpha=\beta+\psi \tilde{f},
$$

where $\psi$ is a new real-valued symmetric bilinear form and $\beta=e^{-\lambda} \alpha_{0}$ is flat. To summarize, we have seen that if $f: M \rightarrow \mathbb{E}^{N} \subset S^{N}$ is a conformal immersion of a locally conformally flat Riemannian manifold with isometric lifting $\tilde{f}: M \rightarrow \mathbb{L}^{N+2}$, then at any given point $p \in M$ the second fundamental form of $\tilde{f}$ decomposes into the sum of a flat symmetric bilinear form $\beta$ and a scalar multiple of $\tilde{f}$.

Just like $\alpha, \beta$ must satisfy the identity

$$
\langle\beta(x, y), \tilde{f}(p)\rangle=-\langle x, y\rangle, \quad \text { for } x, y \in T_{p} M .
$$

Thus it follows from the Gauss equation that the curvature of $M$ is given by

$$
\begin{gathered}
R(x, y, z, w)=\langle\alpha(x, z), \alpha(y, w)\rangle-\langle\alpha(x, w), \alpha(y, z)\rangle \\
=\langle\beta(x, z), \psi(y, w) \tilde{f}(p)\rangle+\langle\psi(x, z) \tilde{f}(p), \beta(y, w)\rangle \\
-\langle\beta(x, w), \psi(y, z) \tilde{f}(p)\rangle-\langle\psi(x, w) \tilde{f}(p), \beta(y, z)\rangle \\
=-\langle x, z\rangle \psi(y, w)-\psi(x, z)\langle y, w\rangle+\langle x, w\rangle \psi(y, z)+\psi(x, w)\langle y, z\rangle .
\end{gathered}
$$

It is not difficult to check that $\psi$ is uniquely determined by the Ricci curvature of $M$ and, in particular, if $M$ has constant sectional curvature $c$, we must have

$$
\psi(x, y)=-\frac{c}{2}\langle x, y\rangle .
$$

An element $x \in T_{p} M$ is regular for $\beta$ if the linear map

$$
\beta(x): T_{p} M \longrightarrow N_{p} M \quad \text { defined by } \quad \beta(x)(y)=\beta(x, y)
$$

has maximal possible rank. It follows from the Lemma in [10 that if $x$ is a regular element of $T_{p} M$ and $n$ is an element of

$$
N(\beta(x))=\left\{n \in T_{p} M: \beta(x)(n)=0\right\},
$$

then

$$
\beta(n)\left(T_{p} M\right) \subset \beta(x)\left(T_{p} M\right) \cap\left[\beta(x)\left(T_{p} M\right)\right]^{\perp},
$$

where the orthogonal complement $(\cdot)^{\perp}$ is taken with respect to the Lorentz inner product in $N_{p} M$.

If $\operatorname{dim} N_{p} M<\operatorname{dim} T_{p} M$, then $N(\beta(x))$ must contain a nonzero element $n$. Since $\beta(n, n) \neq 0, \beta(n, n)$ must be a nonzero null vector in $\beta\left(x, T_{p} M\right) \cap\left[\beta\left(x, T_{p} M\right)\right]^{\perp}$, and hence the Lorentz inner product on $N_{p} M$ must induce a positive semi-definite inner product on $\beta\left(x, T_{p} M\right)$. Since regular elements are dense, we see that the Lorentz inner product must induce a positive semi-definite inner product on

$$
S(\beta)=\operatorname{Span} \text { of } \beta(x, y) \text { for } x, y \in T_{p} M .
$$


Thus $S(\beta)$ must contain a unique lightlike line $L$ which contains the nonzero null vector $\beta(n, n) . L$ in turn contains a unique forward-pointing vector $\eta(p)$ such that

$$
\langle\tilde{f}(p), \eta(p)\rangle=-2 .
$$

It now follows from (13) and the fact that $\beta$ is flat that

$$
\alpha(x, y)=\psi(x, y) \tilde{f}(p)+\phi(x, y) \eta(p)+\beta_{1}(x, y),
$$

where $\phi: T_{p} M \times T_{p} M \rightarrow \mathbb{R}$ is a symmetric bilinear form and $\beta_{1}$ is a flat form which takes its values in the orthogonal complement to the span of $\tilde{f}(p)$ and $\eta(p)$. It follows from (17) and (12) that $\phi(x, y)=(1 / 2)\langle x, y\rangle$, and hence

$$
\alpha(x, y)=\psi(x, y) \tilde{f}(p)+\frac{1}{2}\langle x, y\rangle \eta(p)+\beta_{1}(x, y), \quad \text { for } x, y \in T_{p} M .
$$

If $\operatorname{dim} N_{p} M=\operatorname{dim} T_{p} M$, then just as in the case of isometric immersions (as described more fully in [11]), there are exactly two mutually exclusive possibilities:

Definition. Suppose that $\operatorname{dim} N_{p} M=\operatorname{dim} T_{p} M$. We say that $p$ is a weak umbilic if there is a nonzero element $n \in N(\beta(x))$. We say that $p$ is a nonumbilic if $\beta(x)$ is an isomorphism from $T_{p} M$ to $N_{p} M$.

The case where $p$ is a weak umbilic is exactly like the case in which $\operatorname{dim} N_{p} M<$ $\operatorname{dim} T_{p} M$; we can conclude once again that the second fundamental form can be written in the form (18).

If $p$ is a nonumbilic, it follows from Theorem $2 \mathrm{~b}$ of [1] that there is a basis $\left(e_{n+1}, \ldots, e_{2 n}\right)$ for $N_{p} M$ such that

$$
\left\langle\beta(\cdot, \cdot), e_{\lambda}\right\rangle: T_{p} M \times T_{p} M \longrightarrow R
$$

is a symmetric bilinear form of rank one, for $n+1 \leq \lambda \leq 2 n$, and that

$$
\left\langle e_{\lambda}, e_{\mu}\right\rangle= \begin{cases}1, & \text { if } \lambda=\mu<2 n \\ -1, & \text { if } \lambda=\mu=2 n \\ 0, & \text { if } \lambda \neq \mu .\end{cases}
$$

It follows immediately that we can construct a basis $\left(e_{1}, \ldots, e_{n}\right)$ for $T_{p} M$ so that

$$
\left\langle\beta\left(e_{i}, e_{j}\right), e_{n+k}\right\rangle=0 \quad \text { unless } i=j=k .
$$

According to (14), we can take the basis $\left(e_{1}, \ldots, e_{n}\right)$ to be orthonormal. We agree to set

$$
\beta\left(e_{i}, e_{i}\right)=\frac{1}{x_{i}(p)} e_{n+i},
$$

where we arrange that each $x_{i}(p)$ is a positive number by changing signs of some of the normal vectors $\left(e_{n+1}, \ldots, e_{2 n}\right)$ if necessary. Then

$$
\alpha\left(e_{i}, e_{j}\right)=\delta_{i j} \frac{1}{x_{i}(p)} e_{n+i}+\psi\left(e_{i}, e_{j}\right) \tilde{f}(p),
$$

and we conclude from (12) that

$$
-1=\left\langle\alpha\left(e_{i}, e_{i}\right), \tilde{f}(p)\right\rangle=\frac{1}{x_{i}(p)}\left\langle e_{n+i}, \tilde{f}(p)\right\rangle
$$

and hence that

$$
\tilde{f}(p)=-\sum_{i=1}^{n-1} x_{i}(p) e_{n+i}+x_{n}(p) e_{2 n}
$$


The above facts place significant restrictions on the types of space forms which admit conformal immersions into $\mathbb{E}^{N}$ with low codimension. Indeed, if $M^{n}$ is compact and $u$ is a future-pointing timelike vector in $\mathbb{L}^{N+2}$, the height function

$$
h_{u}: M^{n} \longrightarrow \mathbb{R} \quad \text { defined by } \quad h_{u}(p)=-\langle\tilde{f}(p), u\rangle
$$

must assume its maximum value at some point $p$. At this point, the Hessian

$$
d^{2} h_{u}(p)(x, y)=-\langle\alpha(x, y), u\rangle
$$

must be negative semi-definite. If either $\operatorname{dim} N_{p} M<\operatorname{dim} T_{p} M$ or $\operatorname{dim} N_{p} M=$ $\operatorname{dim} T_{p} M$ and there is a nonzero element in $N(\beta(x))$, it follows from (18) that we can write

$$
d^{2} h_{u}(p)(x, y)=-\psi(x, y)\langle\tilde{f}(p), u\rangle-\frac{1}{2}\langle x, y\rangle\langle\eta(p), u\rangle-\left\langle\beta_{1}(x, y), u\right\rangle .
$$

According to Proposition 1 from [10, there exists a vector $n \in T_{p} M$ such that $\beta_{1}(n, n)=0$, and hence

$$
d^{2} h_{u}(p)(n, n)=-\psi(n, n)\langle\tilde{f}(p), u\rangle-\frac{1}{2}\langle n, n\rangle\langle\eta(p), u\rangle .
$$

Since $\langle\tilde{f}(p), u\rangle$ and $\langle\eta(p), u\rangle$ are both strictly negative, the only way $d^{2} h_{u}(p)$ can be negative definite is for $\psi(n, n)<0$.

If $\operatorname{dim} N_{p} M=\operatorname{dim} T_{p} M$ and $\beta(x)$ is an isomorphism from $T_{p} M$ to $N_{p} M$, then it follows from (20) that $\left\langle e_{2 n}, \tilde{f}(p)\right\rangle<0$. Since $e_{2 n}$ and $u$ are both future-pointing, $\left\langle e_{2 n}, u\right\rangle<0$, and

$$
d^{2} h_{u}(p)\left(e_{2 n}, e_{2 n}\right)=-\frac{1}{x_{n}(p)}\left\langle e_{2 n}, u\right\rangle-\psi\left(e_{n}, e_{n}\right)\langle\tilde{f}(p), u\rangle
$$

can be negative semi-definite only if $\psi\left(e_{n}, e_{n}\right)$ is strictly negative. Thus in either case, it follows from (15) that the conformal structure on $M$ cannot contain a Riemannian metric with constant zero or constant negative sectional curvatures. In particular, $n$-dimensional flat tori and hyperbolic space forms admit no conformal immersions into $\mathbb{E}^{2 n-2}$, a fact that was first pointed out in 13 .

\section{Conformal immersions of Elliptic SPACE FORMS}

We now describe the modifications to our previous argument that are necessary to show that the only $n$-dimensional elliptic space form which admits a conformal immersion in $\mathbb{E}^{2 n-3}$ (or $S^{2 n-3}$ ) is the standard $n$-sphere, thereby completing the proof of the Theorem stated in the introduction.

From the discussion in $\S 4$, we know that a conformal immersion $f: M \rightarrow S^{2 n-3}$ of a manifold $M$ of constant positive curvature lifts to an isometric immersion

$$
\tilde{f}: M \longrightarrow C_{+}^{2 n-2} \subset \mathbb{L}^{2 n-1}
$$

Moreover, since $\operatorname{dim} N_{p} M<\operatorname{dim} T_{p} M$, it follows from (18) that the second fundamental form at $p$ possesses a decomposition

$$
\alpha(x, y)=\langle x, y\rangle e_{n+1}(p)+\beta_{1}(x, y), \quad \text { where } \quad e_{n+1}(p)=\frac{1}{2}[\eta(p)-\tilde{f}(p)] .
$$

We will see in $\S 6$ that the $\operatorname{map} p \mapsto e_{n+1}(p)$ is smooth. Thus we can define a smooth vector bundle $E$ over $M$ whose fiber at $p$ is generated by $F_{*} T_{p} M$ and $e_{n+1}(p)$. Since $e_{n+1}(p)$ is spacelike, the induced fiber metric on $E$ is positive definite, and one checks just like in $\S 2$ that the induced connection is flat. We can let $E^{\perp}$ be 
the orthogonal complement to $e_{n+1}$ in the normal bundle, a bundle with induced metric of Lorentz signature, and define a smooth Gauss map

$$
g: M \longrightarrow G_{n+1}^{+}\left(\mathbb{L}^{2 n-1}\right),
$$

by letting $g(p)$ be the fiber of $E$ at $p$.

Lemma. The differential of the Gauss map $g$ at any point has rank at most $n-3$.

Proof. The argument is almost identical to that presented in $\S 2$. We utilize the index conventions:

$$
1 \leq a, b \leq n ; \quad 1 \leq i, j \leq n+1 ; \quad 1 \leq I, J, K \leq 2 n-1 ; \quad n+2 \leq \lambda, \mu \leq 2 n-1 .
$$

Over a suitable open subset $U$ of $M$, we choose a moving orthonormal frame $\left(e_{1}, \ldots, e_{n}\right)$ for $T M$, and extend our distinguished section $e_{n+1}$ to a moving Lorentz frame $\left(e_{n+1}, \ldots, e_{2 n-1}\right)$ for the normal bundle $N M$, such that

$$
\left(\left\langle e_{I}, e_{J}\right\rangle\right)=\left(\eta_{I J}\right)=\left(\begin{array}{cccc}
1 & \cdot & 0 & 0 \\
\cdot & \cdot & \cdot & \cdot \\
0 & \cdot & 1 & 0 \\
0 & \cdot & 0 & -1
\end{array}\right)
$$

If $\nabla$ denotes the standard connection on $\mathbb{L}^{2 n-1}$, we define the connection forms $\omega_{J}^{I}$ by

$$
\nabla e_{J}=\sum e_{I} \omega_{J}^{I}
$$

The matrix $\left(\omega_{J}^{I}\right)$ takes its values in the Lie algebra of the Lorentz group, or equivalently, the forms $\omega_{I J}=\sum \eta_{I K} \omega_{J}^{K}$ satisfy the skew-symmetry condition $\omega_{J I}=$ $-\omega_{I J}$. We define the canonical one-forms $\theta^{I}$ by $\theta^{a}\left(e_{b}\right)=\delta_{b}^{a}$ and $\theta^{n+1}=\theta_{\lambda}=0$. The connection and canonical forms satisfy a Lorentz version of the structure equations

$$
d \omega_{J}^{I}=-\sum_{K} \omega_{K}^{I} \wedge \omega_{J}^{K}, \quad d \theta^{I}=-\sum_{J} \omega_{J}^{I} \wedge \theta^{J} .
$$

As in $\S 2$, we define

$$
\tilde{\beta}_{1}: T_{p} M \times E_{p} \longrightarrow E_{p}^{\perp} \quad \text { by } \quad \tilde{\beta}\left(x, \sum a_{i} e_{i}\right)=\sum_{\lambda i} a_{i} e_{\lambda} \omega_{\lambda i}(x),
$$

and check that

$$
\left\langle\tilde{\beta}_{1}(x, z), \tilde{\beta}_{1}(y, w)\right\rangle-\left\langle\tilde{\beta}_{1}(x, w), \tilde{\beta}_{1}(y, z)\right\rangle=0, \quad \text { for } x, y \in T_{p} M \text { and } z, w \in E_{p} .
$$

Using a choice of regular element as in $\S 2$, we check that

$$
N\left(\tilde{\beta}_{1}\right)_{p}=\left\{v \in T_{p} M: \omega_{i}^{\lambda}(v)=0\right\}
$$

has dimension at least three. Once again we define an upper semicontinuous function $\nu: M \rightarrow Z$ by $\nu(p)=\operatorname{dim} N\left(\tilde{\beta}_{1}\right)_{p}$, and define an increasing filtration of $M$ by open sets,

$$
U_{0} \subset U_{1} \subset \cdots \subset U_{k} \subset \cdots \subset U_{n}=M, \quad U_{k}=\{p \in M: \nu(p) \leq k\} .
$$

On the open set $U_{k}-\overline{U_{k-1}}, p \mapsto N\left(\tilde{\beta}_{1}\right)_{p}$ is a distribution of constant rank, and it follows from (4) that

$$
d \omega_{i}^{\lambda} \in \text { ideal generated by the } \omega_{j}^{\mu} \text { s; }
$$


so the distribution is involutive. The maximal integral submanifolds form a foliation $\mathcal{F}$ of $U_{k}-\overline{U_{k-1}}$ with $k$-dimensional leaves.

Since the restriction of $\omega_{i}^{\lambda}$ to any leaf of $\mathcal{F}$ is zero, the subbundles $E$ and $E^{\perp}$ are once again preserved by parallel transport along the leaves, and hence the restriction of the Gauss map $g$ to each leaf is constant. Therefore, on the open set $U_{k}-\overline{U_{k-1}}$, the differential of the Gauss map has rank at most $k$. Since $U_{k}$ is empty unless $k \geq 3$, the differential of the Gauss map has rank at most $n-3$ on the union of the sets $U_{k}-\overline{U_{k-1}}$, an open dense subset of $M$. We therefore conclude once again that the differential of $g$ has rank $\leq n-3$ at every point of $M$, and our outline of the proof of the Lemma is complete.

Proof of the Theorem in the case of conformal immersions. With the previous lemma in place, the proof is virtually identical to that presented in $\S 3$.

\section{Smooth Decomposition of the normal BundLe}

Let $V$ and $W$ be finite-dimensional real vector spaces and $\langle\cdot, \cdot\rangle$ a nondegenerate symmetric bilinear form on $W$. A symmetric bilinear map $\beta: V \times V \rightarrow W$ is flat if

$$
\langle\beta(x, z), \beta(y, w)\rangle-\langle\beta(x, w), \beta(y, z)\rangle=0, \quad \text { for } x, y, z, w \in V .
$$

We have seen several examples of flat forms in preceding sections. In this section, we focus on the case in which $\langle\cdot, \cdot\rangle$ has Lorentz signature.

Example 1. For the isometric immersions considered in $\S \S 2$ and 3, we take $V=$ $T_{p} M, W=N_{p} M \oplus \mathbb{R}$ with the Lorentz inner product

$$
\langle\cdot, \cdot\rangle=\langle\cdot, \cdot\rangle_{N_{p} M}-\langle\cdot, \cdot\rangle_{\mathbb{R}}
$$

where $\langle\cdot, \cdot\rangle_{N_{p} M}$ is the usual inner product on $N_{p} M,\langle\cdot, \cdot\rangle_{\mathbb{R}}$ is the usual dot product on $\mathbb{R}$ and

$$
\beta=\alpha \oplus\langle\cdot, \cdot\rangle_{\mathbb{R}} .
$$

It follows immediately from the Gauss equations that $\beta$ is flat.

Example 2. In the case of conformal immersions considered in $\S \S 4$ and 5 , we take $V=T_{p} M, W$ to be the normal space to the isometric immersion $\tilde{f}: M \rightarrow \mathbb{L}^{2 n-1}$ and let

$$
\beta(x, y)=\frac{1}{2}\langle x, y\rangle \eta(p)+\beta_{1}(x, y), \quad \text { for } x, y \in T_{p} M,
$$

the component of the second fundamental form which appears in (13).

Suppose now that $V$ and $W$ are smooth vector bundles over a smooth manifold $M$, that $W$ possesses a smooth Lorentz fiber metric $\langle\cdot, \cdot\rangle$ and that $\beta: V \times V \rightarrow W$ is a smooth map commuting with projections to the base which restricts to a flat symmetric bilinear form $\beta_{p}: V_{p} \times V_{p} \rightarrow W_{p}$, over each $p \in M$. In this case, we say that $\beta: V \times V \rightarrow W$ is a smooth flat form. Let $S\left(\beta_{p}\right)$ denote the subspace of $W_{p}$ generated by $\beta(x, y)$ for all $x, y \in V_{p}$ and let $S\left(\beta_{p}\right)^{\perp}$ denote its orthogonal complement with respect to the Lorentz fiber metric.

Smoothness Lemma. Suppose that $\beta: V \times V \rightarrow W$ is a smooth flat form with $\operatorname{rank}(W) \leq \operatorname{rank}(V)$ and that

1. for each $p \in M, S\left(\beta_{p}\right) \cap S\left(\beta_{p}\right)^{\perp}$ is a one-dimensional subspace $L_{p} \subset W_{p}$,

2. $x \in V_{p}$ and $x \neq 0 \Rightarrow \beta_{p}(x, x) \neq 0$. 
Then the family of lines $p \mapsto L(p)$ varies smoothly with $p$ and defines a smooth rank one subbundle of $W$.

Remark. In the case where $W$ has rank two, the Smoothness Lemma is trivial, while if $W$ has rank three, a special case of the Smoothness Lemma is presented in $\S 2$ of [13, based upon a theorem of Joris (Theorem 1 of [1]) which states that if the square and cube of a real-valued function are smooth, so is the function itself.

In both of the examples described above, the hypotheses of the Smoothness Lemma hold. Indeed, in Example 1, we can take $L_{p}$ to be generated by

$$
\left(e_{n+1}(p), 1\right) \in N_{p} M \oplus \mathbb{R}
$$

while in Example 2, we can take $L_{p}$ to be generated by $\eta(p)$. Thus the Smoothness Lemma implies that the sections $e_{n+1}$ constructed in $\S \S 2$ and 5 are smooth.

Proof of Smoothness Lemma. We can assume that $V$ has a fiber metric and metric connection. Let $p \mapsto e(p)$ be a smoothly varying timelike section of $W$, normalized so that $\langle e(p), e(p)\rangle=-1$. Define a section $\tau: M \rightarrow L$ by requiring that $\langle\tau(p), e(p)\rangle=-1$. It suffices to show that this section is smooth. Locally, $\tau$ can be regarded as a map into $\mathbb{R}^{m}$, where $m=\operatorname{rank} W$.

By a theorem of Boman [2, to show that an $\mathbb{R}^{m}$-valued function on a smooth manifold $M$ is smooth, it suffices to show that its restriction to any smooth curve $\gamma:(a, b) \rightarrow M$ is smooth. Given such a curve $\gamma$, let

$$
U_{k}=\left\{t \in(a, b): \operatorname{dim}\left[S\left(\beta_{\gamma(t)}\right)\right] \geq k\right\} .
$$

We thus obtain a decreasing filtration

$$
(a, b)=U_{0} \supset \cdots \supset U_{k-1} \supset U_{k} \supset \cdots
$$

of open subsets of $(a, b)$. The strategy of proof is to show that $\tau \circ \gamma$ is smooth on $U_{k}$ by negative induction on $k$, the case where $k$ is sufficiently large being trivial, since then $U_{k}$ is empty.

Suppose inductively, therefore, that $\tau \circ \gamma$ is smooth on $U_{k+1}$ and that $t_{0} \in$ $U_{k}-U_{k+1}$. If $t_{0} \in U_{k}-\overline{U_{k+1}}, S\left(\beta_{\gamma(t)}\right)$ is a smoothly varying subspace of $W_{\gamma(t)}$ for $t$ sufficiently close to $t_{0}$. Moreover, the Lorentz inner product $\langle\cdot, \cdot\rangle$ restricts to a degenerate symmetric bilinear form on $S\left(\beta_{\gamma(t)}\right)$ which contains a unique line on which the inner product restricts to zero. This line is $L_{\gamma(t)}$, the fiber of our distinguished line bundle, and must vary smoothly as $S\left(\beta_{\gamma(t)}\right)$ varies smoothly. Thus the vector $\tau(\gamma(t))$ must vary smoothly, for $t$ near $t_{0} \in U_{k}-\overline{U_{k+1}}$.

The only difficulty in the inductive step occurs when $t_{0} \in\left(U_{k} \cap \overline{\left.U_{k+1}\right)}-U_{k+1}\right.$ and the dimension of $S\left(\beta_{\gamma(t)}\right)$ varies in a neighborhood of $t_{0}$. To cover this case, we first choose a parallel moving orthonormal frame $\left(e_{1}, e_{2}, \ldots, e_{n}\right)$ for $V$ along $\gamma$, where $n=\operatorname{rank} V$. Then by definition of $U_{k}$, we can choose indices $i_{1}, \ldots, i_{k}$ and $j_{1}, \ldots, j_{k}$ so that $\beta\left(e_{i_{1}}, e_{j_{1}}\right)(t), \beta\left(e_{i_{2}}, e_{j_{2}}\right)(t), \ldots, \beta\left(e_{i_{k}}, e_{j_{k}}\right)(t)$ are linearly independent for $t=t_{0}$, hence for $t \in\left(t_{0}-\epsilon, t_{0}+\epsilon\right)$, for some positive $\epsilon$. Thus

$$
\beta\left(e_{i_{1}}, e_{j_{1}}\right)(t) \wedge \beta\left(e_{i_{2}}, e_{j_{2}}\right)(t) \wedge \cdots \wedge \beta\left(e_{i_{k}}, e_{j_{k}}\right)(t) \neq 0, \quad \text { for } t \in\left(t_{0}-\epsilon, t_{0}+\epsilon\right) .
$$

Since we are assuming that $t_{0} \notin U_{k+1}$,

$$
\beta\left(e_{i_{1}}, e_{j_{1}}\right)\left(t_{0}\right) \wedge \beta\left(e_{i_{2}}, e_{j_{2}}\right)\left(t_{0}\right) \wedge \cdots \wedge \beta\left(e_{i_{k}}, e_{j_{k}}\right)\left(t_{0}\right) \wedge \beta\left(e_{i}, e_{j}\right)\left(t_{0}\right)=0,
$$

for all choices of indices $i$ and $j$. 
For $i_{k+1}, j_{k+1} \in\{1, \ldots, n\}$ we can then ask the question: does

$$
\beta\left(e_{i_{1}}, e_{j_{1}}\right)(t) \wedge \beta\left(e_{i_{2}}, e_{j_{2}}\right)(t) \wedge \cdots \wedge \beta\left(e_{i_{k}}, e_{j_{k}}\right)(t) \wedge \beta\left(e_{i_{k+1}}, e_{j_{k+1}}\right)(t)
$$

vanish to only finite order at $t_{0}$ ? If so, we can ask whether there exist $i_{k+2}$ and $j_{k+2}$ so that

$$
\beta\left(e_{i_{1}}, e_{j_{1}}\right) \wedge \beta\left(e_{i_{2}}, e_{j_{2}}\right) \wedge \cdots \wedge \beta\left(e_{i_{k}}, e_{j_{k}}\right) \wedge \beta\left(e_{i_{k+1}}, e_{j_{k+1}}\right) \wedge \beta\left(e_{i_{k+2}}, e_{j_{k+2}}\right)(t)
$$

vanishes to only finite order, and so on. The resulting questions can be answered in the affirmative only finitely many times. We finally attain $e_{i_{1}}, \ldots, e_{i_{k+l}}$ and $e_{j_{1}}, \ldots, e_{j_{k+l}}$ so that

$$
\beta\left(e_{i_{1}}, e_{j_{1}}\right) \wedge \cdots \wedge \beta\left(e_{i_{k}}, e_{j_{k}}\right) \wedge \beta\left(e_{i_{k+1}}, e_{j_{k+1}}\right) \wedge \cdots \wedge \beta\left(e_{i_{k+l}}, e_{j_{k+l}}\right)(t)
$$

vanishes to finite order at $t_{0}$, but any further wedge with any $\beta\left(e_{i}, e_{j}\right)$ vanishes to infinite order.

It then follows from the standard proof of Taylor's theorem that when $\epsilon$ is sufficiently small, there is a smooth map

$$
\xi:\left(t_{0}-\epsilon, t_{0}+\epsilon\right) \rightarrow\left\{\text { nonzero decomposable elements in } \Lambda^{k+l}(W)\right\}
$$

such that

$$
\beta\left(e_{i_{1}}, e_{j_{1}}\right) \wedge \cdots \wedge \beta\left(e_{i_{k+l}}, e_{j_{k+l}}\right)(t)=\left(t-t_{0}\right)^{q} \xi(t),
$$

for some positive integer $q$. Let $W^{*}(t)$ be the subspace of $W_{\gamma(t)}$ determined by $\xi(t)$. $W^{*}(t)$ is a smoothly varying subspace of $W_{\gamma(t)}$ for $t$ near $t_{0}$.

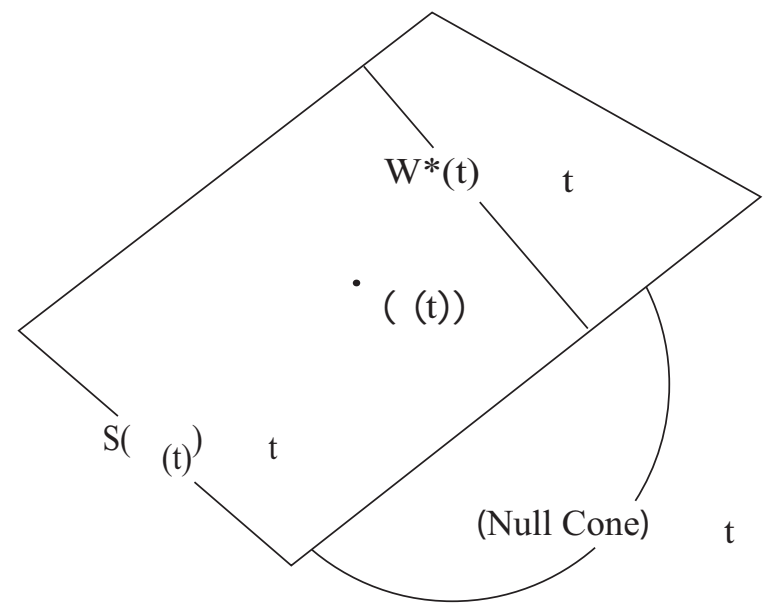

Figure 2. Important subsets of $W \cap \Pi_{t}$. Note that when $t=t_{0}$, the affine space $W^{*}(t) \cap \Pi_{t}$ contains the point $\tau(\gamma(t))$.

It will be helpful to have Figure 2 in mind. At a fixed point $t \in(a, b)$, let $\Pi_{t}$ denote the hyperplane in $W_{\gamma(t)}$ consisting of the vectors $w$ such that $\langle w, e(\gamma(t))\rangle=-1$. Note that the Lorentz inner product $\langle\cdot, \cdot\rangle$ yields a positive-definite flat Riemannian metric on $\Pi_{t}$. The affine subspace $S\left(\beta_{\gamma(t)}\right) \cap \Pi_{t}$ has a dimension which may vary from point to point. Nevertheless, it is always tangent to the intersection of $\Pi_{t}$ 
with the null cone at the point $\tau(\gamma(t))$. For each fixed $t$ we can define a smooth function $f: \Pi_{t} \rightarrow \mathbb{R}$ by

$$
f(w)=[\text { distance from } w \text { to } e(\gamma(t))]^{2},
$$

$e(\gamma(t))$ being the center of the sphere (Null Cone) $\cap \Pi_{t}$. This function restricts to a smooth function $f^{*}: W^{*}(t) \cap \Pi_{t} \rightarrow \mathbb{R}$ which has a unique nondegenerate local minimum, which we denote by $\tau_{k l}(t)$. As $t$ varies, this element $\tau_{k l}(t) \in W^{*}(t) \cap \Pi_{t}$ varies smoothly with $t$; so we obtain a smooth section $\tau_{k l}$ of the vector bundle $\gamma^{*} W$ in a small neighborhood of $t_{0}$.

We claim that $\tau_{k l}-\tau$ vanishes to infinite order at $t_{0}$; in other words, given any $C>0$ and any positive integer $q$,

$$
\left\|\tau_{k l}(t)-\tau(\gamma(t))\right\|<C\left|t-t_{0}\right|^{q}
$$

when $t$ is sufficiently close to $t_{0}$.

To prove the claim, recall that in the terminology of [10, an element $x \in V_{p}$ is called regular if the linear map

$$
\beta_{p}(x): V_{p} \rightarrow W_{p} \quad \text { defined by } \quad \beta_{p}(x)(y)=\beta_{p}(x, y)
$$

has the maximal possible rank for any choice of $x \in V_{p}$. For any fixed choice of $t$, we can choose a regular element $x(t) \in V_{\gamma(t)}$ and an element $n(t)$ in the kernel of $\beta(x(t))$. Note that neither $x(t)$ nor $n(t)$ necessarily depends continuously on $t$. It follows from the Lemma in 10 , that

$$
\beta_{p}(n)\left(V_{p}\right) \subset \beta_{p}(x)\left(V_{p}\right) \cap\left[\beta_{p}(x)\left(V_{p}\right)\right]^{\perp} \subset L_{p},
$$

and by the second hypothesis of the Smoothness Lemma, we can renormalize $n(t)$ so that $\beta_{\gamma(t)}(n(t), n(t))=\tau(\gamma(t))$. We can write

$$
n(t)=f_{1}(t) e_{1}(t)+\cdots+f_{n}(t) e_{n}(t),
$$

where $f_{1}, \ldots, f_{n}$ are bounded real-valued functions, quite possibly discontinuous. If $\tau_{k l}-\tau \circ \gamma$ does not vanish to infinite order, then the angle between $\beta(n(t), n(t))$ and $\xi(t)$ with respect to some positive definite fiber metric on $W$ does not vanish to infinite order. This implies that $\xi(t) \wedge \beta\left(e_{i}(t), e_{j}(t)\right)$ vanishes to only finite order for some $i$ and $j$, and this contradicts maximality of $l$, thus finishing our proof of the claim.

Let $U_{k l}$ be the set of $t_{0} \in U_{k}$ such that either $t_{0} \in U_{k+1}$ or $t_{0} \in U_{k}-U_{k+1}$ and

1. there exists a choice of parallel moving frame of $V$ along $\gamma$ such that for some choice of indices $i_{1}, \ldots, i_{k}$ and $j_{1}, \ldots, j_{k}$,

$$
\beta\left(e_{i_{1}}, e_{j_{1}}\right)\left(t_{0}\right) \wedge \beta\left(e_{i_{2}}, e_{j_{2}}\right)\left(t_{0}\right) \wedge \cdots \wedge \beta\left(e_{i_{k}}, e_{j_{k}}\right)\left(t_{0}\right) \neq 0
$$

2. there is a further choice of indices $i_{k+1}, \ldots, i_{k+l}$ and $j_{k+1}, \ldots, j_{k+l}$ such that

$$
\beta\left(e_{i_{1}}, e_{j_{1}}\right) \wedge \cdots \wedge \beta\left(e_{i_{k}}, e_{j_{k}}\right) \wedge \beta\left(e_{i_{k+1}}, e_{j_{k+1}}\right) \wedge \cdots \wedge \beta\left(e_{i_{k+l}}, e_{j_{k+l}}\right)(t)
$$

vanishes to only finite order at $t_{0}$.

Thus we obtain an inner decreasing filtration by open subsets

$$
U_{k} \supset U_{k 1} \supset \cdots \supset U_{k l} \supset U_{k, l+1} \supset \cdots \supset U_{k+1} .
$$

Assuming that $\tau(\gamma(t))$ is smooth on $U_{k+1}$, we now perform a negative induction on $l$, noting that smoothness is established on $U_{k l}$ when $k+l=\operatorname{rank} W$, because in that case, $\tau(\gamma(t))=\tau_{k l}(t)$. For the inductive step we need to show that if $\tau(\gamma(t))$ is smooth on $U_{k, l+1}$, then it is also smooth on $U_{k l}$. Note that if $t_{0} \in U_{k l}-U_{k, l+1}$, then there is some $\epsilon>0$ such that $\tau_{k l}(t)$ is smoothly defined when $t \in\left(t_{0}-\epsilon, t_{0}+\epsilon\right) \subset U_{k l}$. 
By inductive assumption, $\tau(\gamma(t))$ is smooth for $t \in U_{k, l+1}$ and hence $\tau_{k l}(t)-$ $\tau(\gamma(t))$ is smooth on $U_{k, l+1} \cap\left(t_{0}-\epsilon, t_{0}+\epsilon\right)$. On the other hand, we have proven that $\tau_{k l}(t)-\tau(\gamma(t))$ vanishes to infinite order at any point of $\left(t_{0}-\epsilon, t_{0}+\epsilon\right)-U_{k, l+1}$.

For a given integer $p$, we can set

$$
f(t)= \begin{cases}\left(d^{p} / d t^{p}\right)\left[\tau_{k l}(t)-\tau(\gamma(t))\right], & \text { for } t \in\left(t_{0}-\epsilon, t_{0}+\epsilon\right) \cap U_{k, l+1}, \\ 0, & \text { for } t \in\left(t_{0}-\epsilon, t_{0}+\epsilon\right)-U_{k, l+1}\end{cases}
$$

and

$$
g(t)= \begin{cases}\left(d^{p+1} / d t^{p+1}\right)\left[\tau_{k l}(t)-\tau(\gamma(t))\right], & \text { for } t \in\left(t_{0}-\epsilon, t_{0}+\epsilon\right) \cap U_{k, l+1}, \\ 0, & \text { for } t \in\left(t_{0}-\epsilon, t_{0}+\epsilon\right)-U_{k, l+1}\end{cases}
$$

Both $f$ and $g$ are continuous functions on $\left(t_{0}-\epsilon, t_{0}+\epsilon\right)$ and the argument for Lemma 2 from [1] now implies that $f^{\prime}\left(t_{0}\right)$ exists and equals $g\left(t_{0}\right)$.

In more detail, the assumption that $t_{0} \in U_{k l}-U_{k, l+1}$ implies that given $\epsilon_{1}>0$, there exists a $\delta>0$ such that

$$
\left|t-t_{0}\right|<\delta \Rightarrow|g(t)|<\epsilon_{1}
$$

If $t_{1}$ is the closest point to $t$ in $\left[t_{0}, t\right] \cap\left(U_{k l}-U_{k, l+1}\right)$, then

$$
\left|f(t)-f\left(t_{0}\right)\right|<\epsilon_{1}\left|t-t_{1}\right|<\epsilon_{1}\left|t-t_{0}\right|
$$

so $f$ is differentiable at $t_{0}$ and $f^{\prime}\left(t_{0}\right)=g\left(t_{0}\right)=0$.

By induction on $p$, we thereby conclude that

$$
\frac{d^{p+1}}{d t^{p+1}}[\tau(\gamma(t))]
$$

exists and is continuous at $t_{0}$, for every integer $p$. Since $t_{0}$ was an arbitrary element of $U_{k l}-U_{k, l+1}, \tau(\gamma(t))$ is smooth on $U_{k l}$, completing the inner inductive step. The outer induction step is also completed, and with it the proof of the Smoothness Lemma.

\section{REFERENCES}

[1] I. Amemiya and K. Masuda, On Joris' theorem on differentiability of functions, Kodai Math. J. 12 (1989), 92-97. MR 90a:26005

[2] J. Boman, Differentiability of a function and of its compositions with functions of one variable, Math. Scand. 20 (1967), 249-268. MR 38:6009

[3] J. Cheeger and J. Simons, Differential characters and geometric invariants, Springer Lecture Notes 1167 (1985), 50-80. MR 87g:53059

[4] S. S. Chern, A simple intrinsic proof of the Gauss-Bonnet formula for closed Riemannian manifolds, Annals of Math. 45 (1944), 747-752. MR 6:106a

[5] S. S. Chern and N. Kuiper, Some theorems on the isometric imbedding of compact Riemann manifolds in Euclidean space, Annals of Math. 56 (1952), 422-430. MR 14:408e

[6] S. S. Chern and J. Simons, Characteristic forms and geometric invariants, Annals of Math. 99 (1974), 48-69. MR 50:5811

[7] H. Federer, Geometric measure theory, Springer, New York, 1969. MR 41:1976

[8] S. Kobayashi and K. Nomizu, Foundations of differential geometry (two volumes), John Wiley and Sons, New York, 1963 and 1969. MR 27:2945; MR 38:6501

[9] J. Millson, Examples of nonvanishing Chern-Simons invariants, J. Differential Geometry 10 (1975), 589-600. MR 52:15494

[10] J. D. Moore, Conformally flat submanifolds of Euclidean space, Math. Ann. 225 (1977), 89-97. MR 55:4048

[11] J. D. Moore, Submanifolds of constant positive curvature I, Duke Math. J. 44 (1977), 449484. MR 55:11174] 
[12] J. D. Moore, Codimension two submanifolds of positive curvature, Proc. Amer. Math. Soc. 70 (1978), 72-74. MR 80a:53063

[13] J. D. Moore, On conformal immersions of space forms, Springer Lecture Notes 838 (1981), 203-210. MR 82k:53081

[14] J. D. Moore, On extendability of isometric immersions of spheres, Duke Math. J. 85 (1996), 685-699. MR 97i:53074

[15] M. S. Narasimhan and S. Ramanan, Existence of universal connections, Amer. J. Math. 83 (1961), 563-672. MR 24:A3597

[16] B. O'Neill, Umbilics of constant curvature immersions, Duke Math. J. 32 (1965), 89-97. MR 31:5181

Department of Mathematics, University of California, Santa Barbara, CA 93106

E-mail address: moore@math.ucsb.edu 